\title{
Total knee arthroplasty using trochlear groove as guide for position of femoral component in severe knee osteoarthritis
}

\author{
Gangyong Huang, Jun Xia*, Siqun Wang, Yibing Wei, Jianguo Wu, Feiyan Chen, Jie Chen and Jingsheng Shi
}

\begin{abstract}
Background: Apart from transepicondylar axis, the native femoral sulcus was also reported to be used as a guide for the femoral component position in total knee arthroplasty (TKA). However, it was not shown in patients with severe knee osteoarthritis. This study was conducted to compare the position of trochlear groove in patients with and without osteoarthritis, and to assess whether trochlear groove could be used as a guide for position of femoral component in TKA for severe knee osteoarthritis.

Methods: Total 50 severe knee osteoarthritis patients (Kellgren Lawrence grade 3 or 4) who underwent TKA were included. Meanwhile, 50 patients who underwent arthroscopic surgery without osteoarthritis were included as control. The distance from trochlear groove to the midpoint of a virtual anterior condyle osteotomy line (parallel to the posterior condyle line) (a-b) was recorded by radiological and surgical measurements. Midpoint of transepicondylar axis and trochlear groove were used as guide for placing prosthesis model in TKA, respectively. No-thumb test was performed to assess the patellar tracking. The position of femoral component was finally performed using trochlear groove as guide in TKA.
\end{abstract}

Results: Value of "a-b" was significantly different between osteoarthritic and control knees $(P=0.008)$. During the placement of prosthesis model, similar patellar tracking was detected between using midpoint of transepicondylar axis and trochlear groove as guide $(P>0.05)$. After placing femoral component using trochlear groove as guide, most patients obtained good patellofemoral congruence with pneumatic tourniquet inflated $(n=43)$ or deflated $(n=5)$, and good patellofemoral congruence was also obtained by lateral patellar retinaculum release in two patients.

Conclusion: Despite the shifting of trochlear groove caused by severe knee osteoarthritis, trochlear groove can be used as a guide for position of femoral component, with equivalent patellar tracking compared with transepicondylar axis.

Keywords: Total knee arthroplasty, Knee osteoarthritis, Patellar tracking, Trochlear groove, Prosthesis

\section{Background}

Total knee arthroplasty (TKA) is a well-proven procedure with success rate of more than $90 \%[1,2]$. However, patellofemoral complications (such as anterior knee pain, patellar subluxation, patellofemoral wear and patellar prosthesis loosening and fracture) commonly occurred after TKA, which could lead to poor function of the knee [3]. Previous studies have found that the operative technique, prosthetic design, axial malalignment and malrotation as well as poor patellar tracking contributed to the

\footnotetext{
* Correspondence: dr.xiajun@139.com

Department of Orthopaedic Surgery, Huashan Hospital Fudan University, 12th Wulumuqi Middle Road, Shanghai 200040, China
}

patellofemoral complications [4-7]. Besides, femoral component designs and position were also reported as the main causes of poor patellar tracking in TKA [8-10].

Currently, transepicondylar axis (also known as the surgical epicondylar axis), a line between the medial and lateral epicondyles $\left(3^{\circ}\right.$ of external rotation relative to the femur posterior condyles line), is commonly used as a guide for positioning of the femoral component in TKA [11, 12]. Compared with other reference lines (such as Whiteside's line, femoral transverse axis or posterior condylar axis), the transepicondylar axis was recommended for valgus knees [13-15]. However, it was reported that only $75 \%$ of knees would have been within $3^{\circ}$ of the true epicondylar axis 
when using epicondyles to control rotation, so the clinical estimation of the epicondylar axis was inaccurate [16]. Moreover, transepicondylar axis was just logical and appropriate when used as a principal axis for knees between $0^{\circ}$ and $60^{\circ}$ of flexion [17].

Recently, previous studies have reported that native femoral sulcus could be used as a guide for the femoral component position in TKA $[8,18]$. However, it was not known yet whether trochlear groove was shifting with line of force in severe osteoarthritic knee, and whether the positioning of the femoral component using trochlear groove as reference contributed to the reconstruction of patellar tracking in patients with severe knee osteoarthritis. Thus, this study was conducted to compare the position of trochlear groove in patients with and without osteoarthritis. Meanwhile, we also compared the patellar tracking by using the midpoint of transepicondylar axis and trochlear groove as guide for the position of femoral component. Our study would give the surgeons instruction of the position of femoral component during the TKA for patients with severe knee osteoarthritis.

\section{Methods}

\section{Patients}

Total 197 consecutive patients who underwent selective TKA for knee osteoarthritis in the Department of Orthopaedic Surgery, Shanghai Huashan Hospital from January 2009 to January 2011 were recruited in knee osteoarthritis group for the study. The inclusion criteria were as follows: age of more than 20 years old; a diagnosis of severe knee osteoarthritis (Kellgren Lawrence grade 3 or 4) according to the Kellgren and Lawrence classification criteria [19, 20]; unilateral TKA would be performed. Patient with unwillingness to sign the informed consent form or who had extra-articular malformation was excluded. All osteoarthritis patients who were scheduled for TKA took the fulllength weight-bearing radiographs of both lower extremities and computed tomography (CT) scanning of the knee before surgery. Meanwhile, patients who underwent arthroscopic surgery homochronously in our department were recruited as controls. The patients in control group should meet the following criteria: age of above 20 years old; no history of knee arthropathy; no diagnosis of osteoarthritis through preoperative routine X-radioscopy and arthroscopy; and consent to receive the CT scanning to rule out osteoarthritis. This prospective study was approved by the ethics committee of Shanghai Huashan Hospital.

\section{The CT scanning}

The CT scans were performed using a 256-slice multidetector CT (MDCT) scanner (Philips Brilliance $\mathrm{iCT}$, Philips Healthcare, Cleveland, USA). Patients were placed in supine position with the knee fully extending and feet closed together. All images were reconstructed with 3-mm slice thickness at 3-mm intervals in the transverse (axial) planes. Image data were then transferred to a Centricity picture archiving and communication system (PACS) workstation (Centricity 1.0; GE Healthcare) and analyzed using Centricity PACS software (GE Healthcare, Chalfont St Giles, Buckinghamshire, UK). The first layer image near intercondylar fossa was used and evaluated by two experienced orthopedic radiologists. As shown in Fig. 1a, the lowest point of femoral trochlea (trochlear groove) was defined as point "a", and the midpoint of a virtual anterior condyle osteotomy line (which was parallel to the line of posterior condyle) was defined as point " $\mathrm{b}$ "; the distance from " $a$ " to " $b$ " ( $a-b)$ was measured. The positive value of
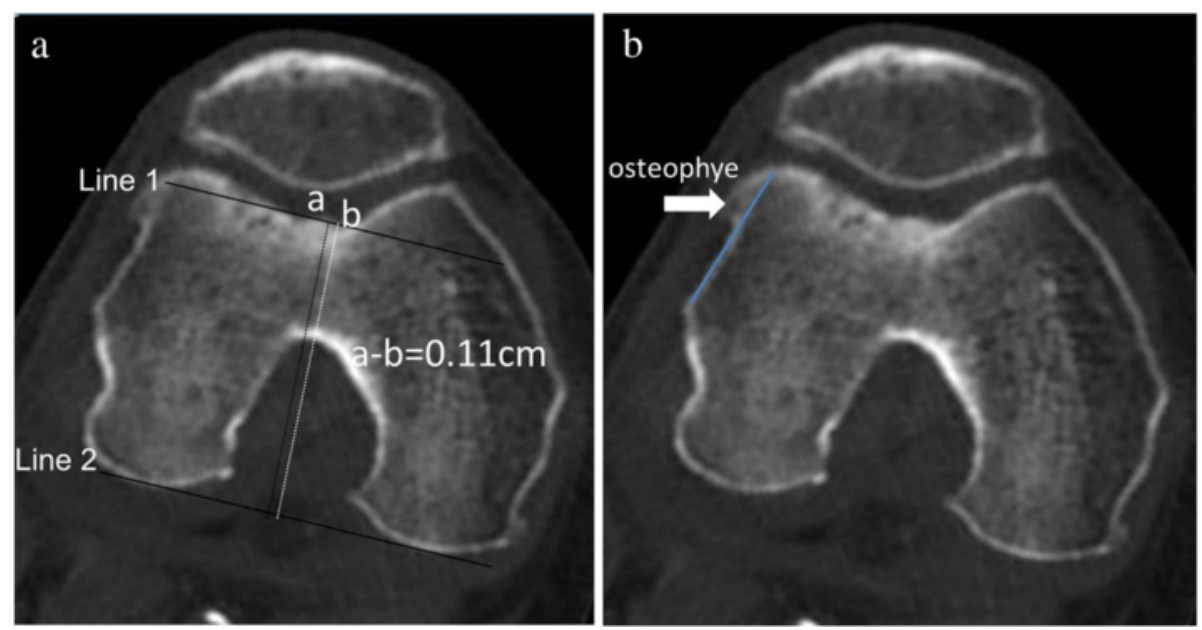

Fig. 1 Preoperative computed tomography (CT) scanning. a the measurement of distances from the lowest point of trochlear groove to the midpoint of anterior condyle in CT images. Line 1: the virtual anterior condyle osteotomy line; line 2: the posterior condyle line. $\mathbf{b}$ the osteophytes in the patients with severe knee osteoarthritis 
distance $\mathrm{a}-\mathrm{b}$ was determined with the "a" point at the lateral of " $\mathrm{b}$ " point; otherwise, the negative value of distance $\mathrm{a}-\mathrm{b}$ was obtained. The measurements for 20 repeated radiographs were performed to determine intra-observer reliability, which were evaluated using an intraclass correlation coefficient (ICC, one way random).

\section{Operative procedure}

All the osteoarthritis patients received knee surface replacement with the preservation of the patella. A senior specialist performed the TKA using Genesis II prosthesis (Genesis II PS High-Flexion, Smith and Nephew, USA) which had an intrinsic $3^{\circ}$ of internal rotation (the medial posterior condyle was thinner than the lateral posterior condyle in this prosthesis). Patients were placed in supine position and a pneumatic tourniquet was inflated on the proximal thigh for reducing intraoperative blood loss. Under general anesthesia, the knee joint was exposed through medial parapatellar arthrotomy. After removing the osteophytes (Fig. 1b) on the femur, the distal femoral osteotomy was performed perpendicular to the mechanical axis of the femur. Then osteotomy for anterior and posterior condyle was performed based on the posterior condyle line. Subsequently, the distance from "a" to " $\mathrm{b}$ " was measured as shown in Fig. 2a. After oblique osteotomy and the preparation of soft tissues, a prosthesis model was placed in each patient using midpoint of transepicondylar axis and trochlear groove as reference point respectively. Meanwhile, patellar tracking was evaluated using no-thumb test [21, 22]. No-thumb test was considered positive if patellar maltracking was identified under tourniquet deflation, otherwise, negative result was considered. Finally, the femoral prostheses were fixed with the trochlear groove as reference point, and patellar tracking was re-evaluated using no-thumb test (Fig. 2b). A lateral retinacular release was performed if no-thumb test was positive.

\section{Postoperative management and follow-up}

Patient were on bed rest with the affected limb elevated at postoperative $24 \mathrm{~h}$ and with ice applied to the wound site to decrease swelling and pain. Intravenous injection of Cefuroxime ( $1.5 \mathrm{~g}$ ) was conducted once during postoperative $12-24 \mathrm{~h}$ for infection prevention. Elastic stocking was used after the removal of negative pressure drainage in case of thrombosis, and the prophylaxis of deep vein thrombosis prophylaxis was also performed by the administration of blood coagulation factor X-antagonist (Rivaroxaban) for 4 weeks. Physiotherapy was performed under the guidance of physiatrician. Patients were encouraged to take quadriceps femoris muscular training and range of motion (ROM) exercises of the knee from postoperative 1 day, and were allowed to take weight-bearing walking with additional protection. Thereafter they were allowed to take independent ambulation and daily living training at postoperative 6 week.

All the patients receiving TKA were followed-up for at least 1 year. During the follow-up, the knee society score (KSS), visual-analogue scale (VAS) score for anterior knee pain and postoperative complications were assessed and recorded.

\section{Statistical analysis}

The sample size was estimated by using the following formula: $\mathrm{N}=\mathrm{Z}^{2} \times(\mathrm{P} \times(1-\mathrm{P})) / \mathrm{E}^{2} \quad[23]$, where $N=$ sample size; $Z=$ the standard normal deviation, usually 1.96 at $95 \%$ confidence level; $P=$ expected prevalence rate of $50 \%$; and $E=$ precision (margin of error at $15 \%$ ). Based on this formula, the minimum sample size needed was 43 , and power of this study was 0.9 .

Data were analyzed using SPSS 15.0. The independent sample $t$-test was applied for the comparison between knee osteoarthritis and control groups. The paired $t$-test

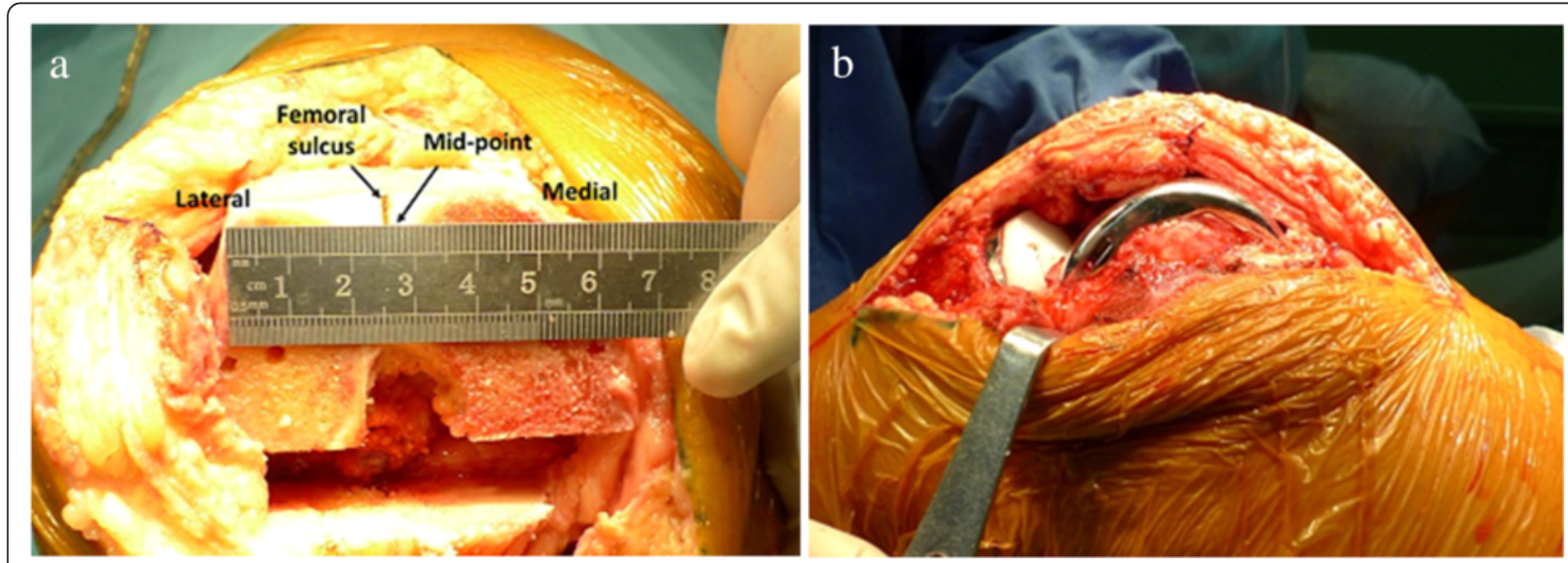

Fig. 2 Operative procedure. a the measurement of distances from the lowest point of trochlear groove to the midpoint of anterior condyle osteotomy line during the surgery. $\mathbf{b}$ patellar tracking was evaluated by no-thumb test 
was used for the comparison of parameters before and after surgery. Comparison of proportions was performed using the $\chi^{2}$ test. The correlation between offset of mechanical axis and position of trochlear groove was assessed using the Pearson's correlation test. For all the analysis, $P<0.05$ was considered statistical significant.

\section{Results}

In this study, only 52 patients with knee osteoarthritis signed the informed consent forms, among whom one patient with rheumatoid arthritis was excluded and the other case accompanied with ipsolateral Crowe-IV developmental dysplasia of the hip were also excluded due to the severe articular valgus. Total 50 patients who underwent arthroscopic surgery homochronously were recruited as controls, including 28 cases of gonarthromeningitis, 17 cases of meniscus injury, 3 cases of knee ligament injury and 2 cases of knee joint pain caused by saphenous nerve entrapment.

Comparison between knee osteoarthritis and control groups As shown in Table 1, knee osteoarthritis and control patients were similar in sex and affected side $(P>005)$. However, patients in knee osteoarthritis group were significantly older than control ones $(P<0.001)$. According to the CT scanning, knee osteoarthritis patients did not differ from control patients in femur anterior condyle width $(P=$ $0.994)$, whereas there was significant difference in the value of " $\mathrm{a}-\mathrm{b}$ " between the two groups $(P=0.008)$. In addition, Fig. 3 showed that the proportion of knee osteoarthritis patients with the value of "a-b" between $-0.10 \mathrm{~cm}$ and $0.00 \mathrm{~cm}$ was the highest, whereas the most control patients had a value of " $\mathrm{a}-\mathrm{b}$ " between $0.00 \mathrm{~cm}$ and $0.05 \mathrm{~cm}$. Meanwhile, the results of ICC showed a significant consistency among the repeated measurements $(r>0.9, P<0.05)$.

\section{Clinical outcomes}

During the operation, the width of femur anterior condyle and the value of "a-b" were re-measured, and similar measurements were obtained compared with CT scanning

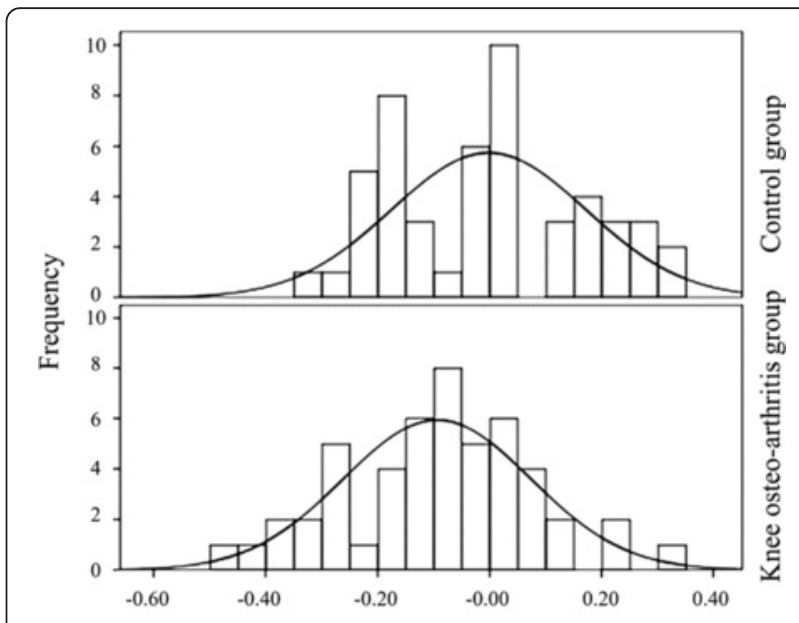

Distance from lowest point of trochlear groove to midpoint of frontal condyle $(\mathrm{a}-\mathrm{b}, \mathrm{cm})$

Fig. 3 Distances from the lowest point of trochlear groove to the midpoint of virtual anterior condyle osteotomy line (a-b) by CT scanning

$(P>0.05$, Table 2 and Fig. 4). In addition, joint deformity was assessed by the full-length weight-bearing radioscopy in these patients with severe knee osteoarthritis. There were $88 \%$ patients $(n=44)$ with varus deformity $\left(10.2 \pm 3.7^{\circ}\right)$ and $12 \%$ patients $(n=6)$ with valgus deformity $\left(6.1 \pm 5.1^{\circ}\right)$. No correlation was found between the intraoperative position of trochlear groove (value of "a-b") and the joint deformity ( $r=0.068, P=0.639)$.

The no-thumb test showed the similar results of patellar tracking by using midpoint of transepicondylar axis and trochlear groove as reference point for placing prosthesis model $(P>0.05$, Table 3$)$. After prostheses placement with trochlear groove as a reference point, total 43 patients had good patellofemoral congruence with the fixation of pneumatic tourniquet. Meanwhile, good patellofemoral congruence was obtained after the deflation of tourniquet in 5 patients. For the other two patients, good patellofemoral congruence was also achieved by lateral retinacular release.

During the median follow up of 2.6 years (range 14.25 years), the median KSS score was 92 (interquartile

Table 1 Comparison between knee osteoarthritis and control group

\begin{tabular}{llll}
\hline Indexes & Knee osteoarthritis group & Control group & $P$-value \\
\hline Sex (male/female) & $16 / 34$ & $24 / 26$ & 0.102 \\
Mean age (range), year & $71.0 \pm 5.8(62-84)$ & $45.5 \pm 12.4(22-65)$ & $<0.001$ \\
Affected side (left/right) & $33 / 17$ & $29 / 21$ & 0.410 \\
Results of CT & & & \\
Width of femur anterior condyle, cm & $5.41 \pm 0.33$ & $5.37 \pm 0.40$ & $95 \%$ Cl: $5.27-5.48$ \\
& $95 \%$ Cl: $5.32-5.52$ & $0.00 \pm 0.17$ & 0.994 \\
Distance from "a" to "b" (a-b), cm & $-0.09 \pm 0.17$ & $95 \%$ Cl: $-0.47-0.52$ & 0.008 \\
\end{tabular}

Data of continuous variables were shown as mean \pm SD; Data of categorical variables were presented as proportions. CT: computed tomography. Distance a-b was the distance from the lowest point of trochlear groove to the midpoint of virtual anterior condyle osteotomy line 
Table 2 Measurements of femur anterior condyle width and distance from "a" to "b" by CT scanning and intraoperative measurement

\begin{tabular}{llll}
\hline Indexes & CT measurement & Intraoperative measurement & $P$-value \\
\hline Width of femur anterior condyle, cm & $5.41 \pm 0.33$ & $5.43 \pm 0.43$ & 0.200 \\
Distance from "a" to "b" (a-b), cm & $95 \%$ Cl: $5.32-5.52$ & $95 \%$ Cl: $5.31-5.55$ & $0.05 \pm 0.15$ \\
& $-0.09 \pm 0.17$ & $95 \%$ Cl: $0.01-0.09$ \\
\hline
\end{tabular}

Distance $a-b$ was the distance from the lowest point of trochlear groove to the midpoint of anterior condyle osteotomy line

range: $80-104)$. Total $90 \%$ (45/50) patients had VAS score lower than 3. Among other 5 patients, one patient had VAS score of 4 and four patients had VAS score of 3 . One patient developed deep vein thrombosis of lower limb at 7 days and 3 weeks after surgery and was cured by conventional treatment. No other complications, such as pulmonary embolism, postoperative periprosthetic fracture, wound infection and flexion instability from malrotation, occurred in these patients during follow up.

\section{Discussions}

It has been reported that trochlear groove was near to the midline of the medial and lateral condyles in normal knees and it would be moved during the flexion of joint [24, 25]. In the present study, trochlear groove was shown at the midpoint of a virtual anterior condyle osteotomy line (which was parallel to the posterior condyle line) of knee without osteoarthritis but was $0.9 \mathrm{~mm}$ medial to that midpoint in osteoarthritic knee. However, Lingaraj and Bartlett (2009) reported that the mean sulcus position was $0.7 \mathrm{~mm}$ lateral to the midline of the distal femoral resection in patients undergoing TKA [26]. Besides, a previous study by Eckhoff et al. (1996) demonstrated that the sulcus of the

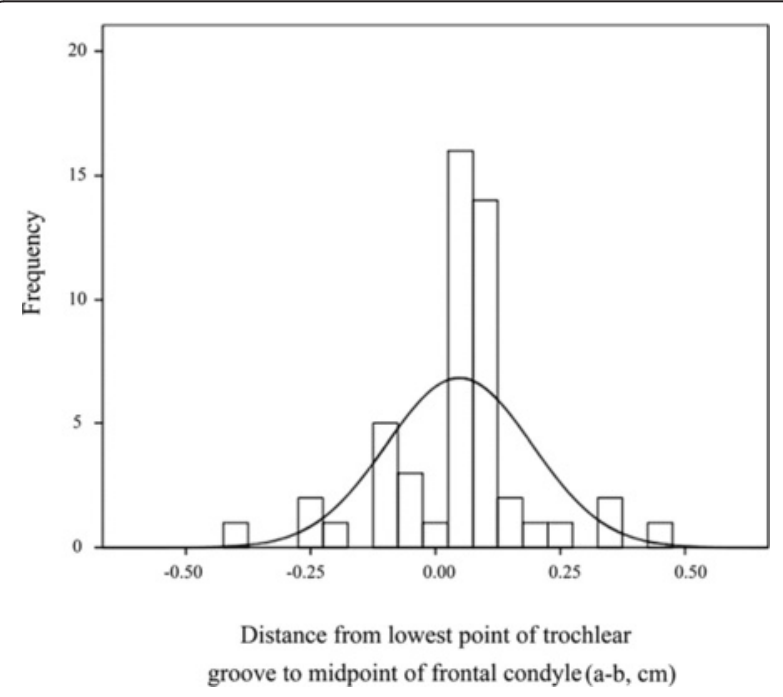

Fig. 4 Measured distances from the lowest point of trochlear groove to the midpoint of anterior condyle osteotomy line (a-b) during the surgery trochlear groove was also lateral to the midline but with no significant difference between osteoarthritic and normal knees [25]. The inconsistent results might be explained by the different reference line used as the midline of distal femur for the position of trochlear groove, as Lingaraj and Bartlett used the midline perpendicular to the distal margin of oblique osteotomy [26], Eckhoff et al. used the midline perpendicular to the posterior condyle line [25], and our study adopted the midline perpendicular to the distal margin of anterior condyle osteotomy. Besides, the severity of knee osteoarthritis causing joint deformity might be the other reason for the different results among the aforementioned studies. Anyhow, it indicated that the trochlear groove was significantly shifted during the occurrence of knee osteoarthritis. On the other hand, there was no significant difference in the distance $\mathrm{a}-\mathrm{b}$ by using CT scanning and intraoperative measurement, suggesting that the position of trochlear groove using the distal margin of anterior condyle osteotomy as reference was stable. However, considering CT scan could not support full visualization of cartilage [27] and might produce errors between radiological and clinical determination of trochlear groove, further study was needed to verify the shift of trochlear groove in osteoarthritis and explore the mechanism.

Patients with severe knee osteoarthritis were always accompanied with varus or valgus deformity [28]. In this study, most of patients (88\%) had varus deformity and $12 \%$ patients had valgus deformity; nevertheless, no significant correlation was found between the position of trochlear groove and the joint deformity. This was in consistent with Lingaraj and Bartlett (2009) who also found the difference in the mean sulcus position was not significant between valgus and varus knees [26].

During the surgery, the no-thumb test showed the similar patellar maltracking by using midpoint of transepicondylar axis and trochlear groove as guide for placing prosthesis model. Previous studies have showed that the position of

Table 3 Results of no-thumb test

\begin{tabular}{lllll}
\hline \multirow{2}{*}{$\begin{array}{l}\text { Guide for position of femoral } \\
\text { prosthesis }\end{array}$} & \multicolumn{2}{l}{ Results of no-thumb test } & P-value \\
\cline { 2 - 3 } & positive & negative & \\
\hline Midpoint of transepicondylar axis & 5 & 45 & 0.436 \\
Trochlear groove & 2 & 48 & \\
\hline
\end{tabular}


femoral prosthesis could influence the patellar tracking $[10,18]$. Also for the patellar prosthesis, it was reported that about $2.5 \mathrm{~mm}$ inward shifting of patellar prosthesis could improve patellar tracking [29]. In this study, TKA was performed with the preservation of the patella. Thus, the patellar tracking was mainly affected by the position and design of femoral prosthesis as well as soft tissue technique. Furthermore, it was reported that the patellar tracking was more associated with the changed position of trochlear groove in the TKA without patellar replacement [30-32]. This further supported that trochlear groove might be the most appropriate guide for position of femoral prosthesis in this study. In addition, it has been reported the slightly external rotation of femoral prosthesis was useful for improving patellar tracking in TKA [33-36]. However, when a conventional femoral component was externally rotated, some rotational incongruity may occur between the femoral and tibial articular surfaces regardless of the position of the tibial component [9]. Thus the use of a femoral component with a thicker posterolateral than posteromedial femoral condyle would minimize the rotational malalignment [37]. Despite the neutral rotation of the femoral component, good patellar tracking was also achieved after TKA by using trochlear groove as a reference.

In addition, previous study has reported that trochlear groove was not a straight line [38]. In this study, only the lowest point of trochlear groove at the distal margin of anterior condyle osteotomy (which was available for positioning) was used in this study due to the limitation of operation. Thus, more studies were required to verify the results of this study.

There were some other limitations in this study. Firstly, patients aged over 70 years old, who underwent arthroscopy but not had knee osteoarthritis, were rare, so there was a big difference in mean age of knee osteoarthritis and control groups in this study, which would affect the results. Secondly, patellar tracking after the placement of prosthesis model was only assessed using no-thumb test, and the patellar maltracking might be overestimated [39]. Third, prostheses were finally fixed with the trochlear groove as reference, and comparison of patellar tracking after the placement of prosthesis by using transepicondylar line and trochlear groove as references was not performed. Besides, most of patients $(88 \%)$ in this study had varus deformity, which may bring bias for the results of this study. Hence, further prospective randomized control study should be performed to compare the clinical outcomes of TKA using transepicondylar axis and trochlear groove as reference for knee osteoarthritis patients, as well as to confirm the accuracy of the femoral component placement using trochlear groove.

\section{Conclusions}

In conclusion, it was found that trochlear groove was shifted during the occurrence of severe osteoarthritis. Similar patellar tracking was detected by no-thumb test when using trochlear groove and transepicondylar axis as guide for position of femoral component. Meanwhile, no correlation was found between position of trochlear groove and joint deformity. Our study would give the surgeons a reference that trochlear groove could be used as a guide for position of femoral component during the TKA for patients with severe knee osteoarthritis.

\section{Abbreviations}

TKA, total knee arthroplasty; CT, computed tomography; MDCT, multi-detector CT; PACS, picture archiving and communication system; ICC, intraclass correlation coefficient; ROM, range of motion; KSS, knee society score; VAS, visual-analogue scale.

\section{Acknowledgements \\ None. No fund supporting was obtained for this article.}

Availability of data and materials

The datasets supporting the conclusions of this article are included within the article.

\section{Authors' contributions}

GH and JX participated in the design of this study, and they both performed the statistical analysis. SW, YW, JW and FC carried out the study and collected important background information. JC and JS drafted the manuscript. All authors read and approved the final manuscript.

\section{Competing interests}

All the author announce that there are no conflict interests and consent to publish this article.

\section{Ethics approval and patients' consent}

This prospective study was approved by the ethics committee of Shanghai Huashan Hospital. All the participants in the study signed the informed consent forms.

Received: 4 January 2016 Accepted: 30 April 2016

Published online: 23 May 2016

\section{References}

1. Choong PF, Dowsey MM, Stoney JD. Does accurate anatomical alignment result in better function and quality of life? Comparing conventional and computer-assisted total knee arthroplasty. J Arthroplasty. 2009;24:560-9.

2. Popa MA, Wera GD, Goldberg VM. Surgical Management of the Unstable Total Knee Arthroplasty. American Academy of Orthopaedic Surgeons, AAOS Now. 2014

3. Russell R, Huo M, Jones R. Avoiding patellar complications in total knee replacement. Bone Joint J. 2014;96:84-6.

4. Meijerink HJ. Clinical and mechanical assessment of total knee arthroplasty: a multifactorial approach. [SI: sn]; 2012.

5. Meftah M, Jhurani A, Bhat JA, Ranawat AS, Ranawat CS. The effect of patellar replacement technique on patellofemoral complications and anterior knee pain. J Arthroplasty. 2012;27:1075-80.

6. Vanbiervliet J, Bellemans J, Verlinden C, Luyckx J-P, Labey L, Innocenti B, et al. The influence of malrotation and femoral component material on patellofemoral wear during gait. J Bone Joint Surg (Br). 2011;93:1348-54.

7. Meijerink H, Barink M, van Loon C, Schwering P, Donk R, de Waal Malefijt NVM. The trochlea is medialized by total knee artroplasty. Clinical and Mechanical Assessment of Total Knee Arthroplasty. 2012;78:55

8. Chiu F-Y. Native femoral sulcus as a guide for the position of the femoral component in primary total knee arthroplasty. Knee Surg Sport Tr A. 2006;14:437-42. 
9. Kong C-G, Park S-W, Yang H, In Y. The effect of femoral component design on patellar tracking in total knee arthroplasty: Genesis II prosthesis versus Vanguard prosthesis. Arch Orthop Trauma Surg. 2014;134:571-6.

10. Rasmussen G. The Effect of Greater External Rotation of the Femoral Component as a Result of Gap Ligament Balancing on Patellar Tracking and Flexion Instability in Total Knee Arthroplasty. J Bone Joint Surg. 2012;94:194.

11. Aglietti P, Sensi L, Cuomo P, Ciardullo A. Rotational position of femoral and tibial components in TKA using the femoral transepicondylar axis. Clin Orthop Relat Res. 2008;466:2751-5.

12. Hutter EE, Granger JF, Beal MD, Siston RA. Is there a gold standard for TKA tibial component rotational alignment? Clin Orthop Relat Res. 2013;471:1646-53.

13. Seo J-G, Moon Y-W, Lim J-S, Park S-J, Kim S-M. Mechanical axis-derived femoral component rotation in extramedullary total knee arthroplasty: a comparison between femoral transverse axis and transepicondylar axis. Knee Surg Sports Traumatol Arthrosc. 2012;20:538-45.

14. Olcott CW, Scott RD. A comparison of 4 intraoperative methods to determine femoral component rotation during total knee arthroplasty. J Arthroplasty. 2000;15:22-6.

15. Newbern DG, Faris PM, Ritter MA, Keating EM, Meding JB, Berend ME. A clinical comparison of patellar tracking using the transepicondylar axis and the posterior condylar axis. J Arthroplasty. 2006;21:1141-6.

16. Kinzel V, Ledger M, Shakespeare D. Can the epicondylar axis be defined accurately in total knee arthroplasty? Knee. 2005;12:293-6.

17. Kobayashi H, Akamatsu Y, Kumagai K, Kusayama Y, Aratake M, Saito T. Is the surgical epicondylar axis the center of rotation in the osteoarthritic knee? J Arthroplasty. 2015;30:479-83.

18. Chiu F-Y. The native femoral sulcus as the guide for the medial/lateral position of the femoral component in knee arthroplasty: Normal patellar tracking in 690/700 knees - a prospective evaluation. Acta Orthop. 2006;77:501-4.

19. Steadman JR, Briggs KK, Matheny LM, Ellis HB. Ten-year survivorship after knee arthroscopy in patients with Kellgren-Lawrence grade 3 and grade 4 osteoarthritis of the knee. Arthroscopy. 2013;29:220-5.

20. Schiphof D, de Klerk BM, Kerkhof HJ, Hofman A, Koes BW, Boers M, et al. Impact of different descriptions of the Kellgren and Lawrence classification criteria on the diagnosis of knee osteoarthritis. Ann Rheum Dis. 2011;70:1422-7.

21. Cho W-S, Woo J-H, Park H-Y, Youm Y-S, Kim B-K. Should the 'no thumb technique'be the golden standard for evaluating patellar tracking in total knee arthroplasty? Knee. 2011;18:177-9.

22. Kim JH, Choi $\mathrm{CH}$, Koo MH, Choi WS. A study on the criteria for lateral retinacular release in total knee arthroplasty: the no thumb technique with one stitch method. J Korean Knee Soc. 2008;20:123-8.

23. Araoye MO. Research methodology with statistics for health and social sciences. Ilorin: Nathadex Publishers; 2003. 118.

24. Shih Y-F, Bull AM, Amis AA. The cartilaginous and osseous geometry of the femoral trochlear groove. Knee Surg Sport Tr A. 2004;12:300-6.

25. Eckhoff DG, Montgomery WK, Stamm ER, Kilcoyne RF. Location of the femoral sulcus in the osteoarthritic knee. J Arthroplasty. 1996;11:163-5.

26. Lingaraj K, Bartlett J. The femoral sulcus in total knee arthroplasty. Knee Surg Sport Tr A. 2009;17:499-502.

27. Kazam JK, Nazarian LN, Miller TT, Sofka CM, Parker L, Adler RS. Sonographic evaluation of femoral trochlear cartilage in patients with knee pain. J Ultras Med. 2011;30:797-802.

28. Sharma L, Song J, Dunlop D, Felson D, Lewis CE, Segal N, et al. Varus and valgus alignment and incident and progressive knee osteoarthritis. Ann Rheum Dis. 2010;69:1940-5.

29. Anglin C, Brimacombe JM, Wilson DR, Masri BA, Greidanus NV, Tonetti J, et al. Biomechanical consequences of patellar component medialization in total knee arthroplasty. J Arthroplasty. 2010;25:793-802.

30. Barink M, Meijerink H, Verdonschot N, van Kampen A, de Waal Malefijt M. Asymmetrical total knee arthroplasty does not improve patella tracking: a study without patella resurfacing. Knee Surg Sport Tr A. 2007;15:184-91.

31. Tanzer M, McLean CA, Laxer E, Casey J, Ahmed AM. Effect of femoral component designs on the contact and tracking characteristics of the unresurfaced patella in total knee arthroplasty. Can J Surg. 2001;44:127.

32. Ostermeier S, Buhrmester O, Hurschler C, Stukenborg-Colsman C. Dynamic in vitro measurement of patellar movement after total knee arthroplasty: an in vitro study. BMC Musculoskel Dis. 2005;6:30.

33. Barink M, Van de Groes S, Verdonschot N, de Waal Malefijt M. The trochlea is bilinear and oriented medially. Clin Orthop Relat R. 2003:411:288-95.
34. Varadarajan KM, Rubash HE, Li G. Are current total knee arthroplasty implants designed to restore normal trochlear groove anatomy? J Arthroplasty. 2011;26:274-81.

35. Barink M, Van de Groes S, Verdonschot N, Malefijt MDW. The difference in trochlear orientation between the natural knee and current prosthetic knee designs; towards a truly physiological prosthetic groove orientation. J Biomech. 2006;39:1708-15.

36. Matsuda S, Miura H, Nagamine R, Urabe K, Hirata G, Iwamoto Y. Effect of femoral and tibial component position on patellar tracking following total knee arthroplasty: 10-year follow-up of Miller-Galante I knees. Am J Knee Surg. 2000;14:152-6.

37. Bourne R, Laskin RS, Guerin J. Ten-year results of the first 100 Genesis II total knee replacement procedures. Orthopedics. 2007:30:83-5.

38. Feinstein WK, Noble PC, Kamaric E, Tullos HS. Anatomic alignment of the patellar groove. Clin Orthop Relat R. 1996:331:64-73.

39. Kim JH, Lee S, Ko DO, Yoo CW, Chun TH, Lee JS. The analysis of risk factors in No thumb test in total knee arthroplasty. Clin Orthop Surg. 2011:3:274-8.

\section{Submit your next manuscript to BioMed Central and we will help you at every step:}

- We accept pre-submission inquiries

- Our selector tool helps you to find the most relevant journal

- We provide round the clock customer support

- Convenient online submission

- Thorough peer review

- Inclusion in PubMed and all major indexing services

- Maximum visibility for your research

Submit your manuscript at www.biomedcentral.com/submit
Biomed Central 